\title{
A common mutation and a novel mutation in Japanese patients with van der Knaap disease
}

Received: 8 April 2003 / Accepted: 10 September 2003/Published online: 13 November 2003

(C) The Japan Society of Human Genetics and Springer-Verlag 2003

\begin{abstract}
Van der Knaap disease, or megalencephalic leukoencephalopathy with subcortical cysts (MLC), is an autosomal recessive disorder clinically characterized by macrocephaly, ataxia, spasticity, and mental decline. Magnetic resonance imaging (MRI) shows swollen brain with diffuse white-matter abnormalities and subcortical cysts, particularly in the anterior-temporal region. Recently, the $M L C 1$ gene was identified as the gene responsible for this disorder, and mutations in this gene were described in several patients. We studied three Japanese patients with van der Knaap disease at the molecular genetic level. Two of them were homozygous for a previously-described mutation, S93L, and one was a compound heterozygote for S93L and a novel mutation, $452-468 \mathrm{del}+\mathrm{g}$,
\end{abstract}

S. Tsujino $(\bowtie) \cdot$ N. Kanazawa

Department of Inherited Metabolic Disease,

National Institute of Neuroscience,

National Center of Neurology and Psychiatry,

4-1-1 Ogawahigashi, Kodaira,

Tokyo 187-8502, Japan

E-mail: tsujino@ncnp.go.jp

Tel.: + 81-42-3412711

Fax: + 81-42-3461746

H. Yoneyama

Department of Pediatrics,

Kitakyushu Rehabilitation Center for Children with Disabilities,

Kitakyushu, Japan

M. Shimono · A. Kawakami

Department of Pediatrics,

University of Occupational and Environmental Health,

Kitakyushu, Japan

Y. Hatanaka $\cdot$ T. Shimizu

Department of Neurology,

Teikyo University School of Medicine,

Tokyo, Japan

H. Oba

Department of Radiology,

Teikyo University School of Medicine,

Tokyo, Japan which leads to frameshift with a premature termination codon. Combining our data with previous reports allowed us to estimate the molecular genetic basis of this disorder in seven Japanese patients. In summary, S93L was observed in six of seven $(85.7 \%)$ patients at least in one allele, and ten of $14(71.4 \%)$ alleles had this mutation. Therefore, S93L appears to be fairly frequent in Japanese patients with van der Knaap disease, and analysis for this mutation in DNA isolated from leukocytes would provide for an easy and precise diagnosis of this disorder in Japanese patients.

Keywords van der Knaap disease $\cdot$ Megalencephalic leukoencephalopathy $\cdot$ Subcortical cysts $\cdot$ MLC ·

$M L C 1 \cdot$ Mutation - Japanese

\section{Introduction}

Megalencephalic leukoencephalopathy with subcortical cysts (MLC; MIM604004), also called van der Knaap disease, is an autosomal recessive disorder characterized by macrocephaly, delayed onset of deterioration of motor functions with ataxia and spasticity, and mental decline (van der Knaap et al. 1995). Magnetic resonance imaging (MRI) shows swollen brain with diffuse whitematter abnormalities and subcortical cysts, particularly in the anterior-temporal region. A histopathological study revealed spongiform leukoencephalopathy without cortical involvement in this disorder (van der Knaap et al. 1996). A gene locus of this disorder was assigned on chromosome $22 \mathrm{q}_{\text {tel }}$ (Topçu et al. 2000), the responsible gene, $M L C 1$, was identified in the locus, and mutations in this gene were described in several patients (Leegwater et al. 2001). The function of the protein encoded by $M L C l$ is not yet known, but it is speculated to be a membrane protein with eight predicted transmembrane domains (Leegwater et al. 2001).

In this report, we have analyzed the $M L C 1$ gene of three additional Japanese patients with van der Knaap 
disease and found a novel mutation and observed that $\mathrm{S} 93 \mathrm{~L}$ is quite common in Japanese patients.

\section{Materials and methods}

Patients and screening for $\mathrm{S} 93 \mathrm{~L}$

All three patients studied are Japanese and apparently unrelated. Their clinical information is summarized in Table 1, and their MRIs are shown in Fig. 1. After informed consent was obtained from the parents of all three patients, peripheral leukocytes were collected. Initially, in order to detect a previously-described mutation, S93L (Leegwater et al. 2001), genomic DNA was isolated from peripheral leukocytes, and a genomic PCR fragment containing exon 4 was amplified by PCR with intronic primers 1 (5'-TTCAGATCCTTCTGGAAGCG-3') and 2 (5'-ACACTGTC TGTCAGCCCCTC-3'). The PCR fragment was digested with the restriction endonuclease Sty I (New England Biolabs, Inc., Beverly, MA, USA), and electrophoresed through 3.0\% NuSieve agarose (BMA, Rockland, ME, USA). The PCR product (210 bp) was cleaved into two segments (106 bp and $104 \mathrm{bp}$ ) in the presence of this nucleotide change, 393C $>$ T (S93L), whereas the wild-type PCR product was not cleaved. The results were also confirmed by direct sequencing using the same primers.

\section{Sequencing of the $M L C 1$ cDNA}

In the case of a patient who was not homozygous for S93L, then, total RNA was extracted from peripheral leukocytes using the Isogen kit (Nippon Gene, Tokyo, Japan). First-strand cDNA fragment synthesis was performed using oligo dT and the Superscript Preamplification System (Life Technologies, Rockville, MD, USA) following the manufacturer's specifications. Three DNA fragments encompassing the entire coding region of the $M L C 1$ cDNA were amplified by PCR using primers 3 (5'-ACACGTGGCTGTACATTCAG-3') and 4 (5'-TGGGTTCAGGACTAGTTTGC-3') for fragment 1 , primers 5 (5'-ACGCCAATGTGATTCCCAAC-3') and 6 (5'-AGACGTGAGGCTGCTTATGG-3') for fragment 2, and primers 7 (5'-TGCCATTGCCAGTCATGTGG-3') and 8 (5'TTGTGCGTTTCCATGCTTGG-3') for fragment 3. Our standard PCR conditions were: 30 cycles of denaturation at $94^{\circ} \mathrm{C}$ for $1 \mathrm{~min}$; annealing at $65^{\circ} \mathrm{C}$ for $1 \mathrm{~min}$; extension at $72^{\circ} \mathrm{C}$ for $2 \mathrm{~min}$, and an additional extension at $72^{\circ} \mathrm{C}$ for $10 \mathrm{~min}$. The PCR products were separated by electrophoresis through $1.5 \%$ agarose gels, purified by GeneElute Agarose Spin Column (Sigma, St Louis, MO, USA), and directly sequenced using the BigDye Terminator Cycle Sequencing FS Ready Reaction kit using ABI Prism 310 Genetic Analyzer (Applied Biosystems, Foster City, CA, USA) according to manufacturer's instructions.
Fig. 1a-f The brain MRI findings of the three patients. Patient 1 (a and b): a $T_{2}$-weighted axial image shows diffuse hyperintensity in cerebral white matter (including subcortical U-fibers). b $\mathrm{T}_{1}$ weighted paramidsagittal image shows subcortical cysts in frontoparietal region. Patient 2 (c and d): $\mathbf{c} \mathrm{T}_{2}$-weighted axial image demonstrates marked dilatation of lateral ventricles and diffuse hyperintensity in cerebral white matters. d FLAIR coronal image shows diffuse hyperintensity in cerebral white matter and focal hypointensity in the white matter of right inferior frontal gyrus, indicating a subcortical cyst. Patient 3 (e and $\mathbf{f}$ ): $\mathbf{e} \mathrm{T}_{2}$-weighted axial image shows macrocephaly and diffuse hyperintensity in cerebral white matters (including subcortical U-fibers). f FLAIR axial image shows diffuse hyperintensity of cerebral white matters and enlarged gyri accompanied with subcortical cyst in right temporal tip

Sequencing of the allele not having S93L

To determine the sequence of the other allele to that with $\mathrm{S} 93 \mathrm{~L}$, primer 9 (5'-TCATTCCAGTGCATCCCTTC-3') was designed, where underlined $\mathrm{C}$ is complementary only to the sequence of the wild-type (without $\mathrm{S} 93 \mathrm{~L}$ ) allele and underlined $\mathrm{T}$ is mismatched exclusively to avoid misannealing to the allele. A genomic PCR fragment amplified using this primer was sequenced.

\section{Results}

Screening the three patients for S93L $(393 \mathrm{C}>\mathrm{T})$ using PCR-RFLP revealed that two (patients 2 and 3 ) were homozygous and one (patient 1) was heterozygous for S93L (Fig. 2). To elucidate the other allele of patient 1, the $M L C 1$ cDNA fragments were amplified by RT-PCR from leukocyte RNA of the patient and directly sequenced. The sequence was ambiguous and suggested the presence of a deletion or insertion in the other allele. Thus, we amplified only the unknown allele by genomic PCR using primer 9, which anneals to the allele not having S93L (see "Materials and methods"). As a result, we found a novel mutation, a deletion of 17-bp nucleotides, and a retention of one nucleotide $g$ (452$468 \mathrm{del}+\mathrm{g})($ Fig. 3a). No other nucleotide difference was found in the entire coding region of the cDNA between the patient and a control. Therefore, the patient was compound heterozygous for S93L and $452-468 \mathrm{del}+\mathrm{g}$. StyI digestion of a cDNA fragment amplified by

Table 1 Summary of the clinical information of the tree studied patients

\begin{tabular}{|c|c|c|c|c|c|c|c|c|c|c|}
\hline Patient & Gender & $\begin{array}{l}\text { Present } \\
\text { age }\end{array}$ & $\begin{array}{l}\text { Consanguinity } \\
\text { of parents }\end{array}$ & $\begin{array}{l}\text { Head } \\
\text { circumference }\end{array}$ & $\begin{array}{l}\text { Initial } \\
\text { mental } \\
\text { and motor } \\
\text { development }\end{array}$ & Spasticity & Ataxia & Seizures & $\begin{array}{l}\text { Present } \\
\text { intellectual } \\
\text { function }\end{array}$ & $\begin{array}{l}\text { Genetic } \\
\text { mutation }\end{array}$ \\
\hline 1 & Female & 23 & - & $\begin{array}{l}56.3 \mathrm{~cm}(+6.5 \mathrm{SD}), \\
2 \mathrm{yrs} ; \\
66.4 \mathrm{~cm}(+10.8 \mathrm{SD}), \\
23 \mathrm{yrs}\end{array}$ & Normal & + & + & + & Deterioration & $\begin{array}{l}\text { S93L/452 } \\
-468 \mathrm{del}+\mathrm{g}\end{array}$ \\
\hline 3 & Male & 32 & $\begin{array}{l}\text { First } \\
\text { cousins }\end{array}$ & $\begin{array}{l}63 \mathrm{~cm}(+5.5 \mathrm{SD}), \\
32 \mathrm{yrs}\end{array}$ & Normal & + & + & + & Deterioration & S93L/S93L \\
\hline
\end{tabular}



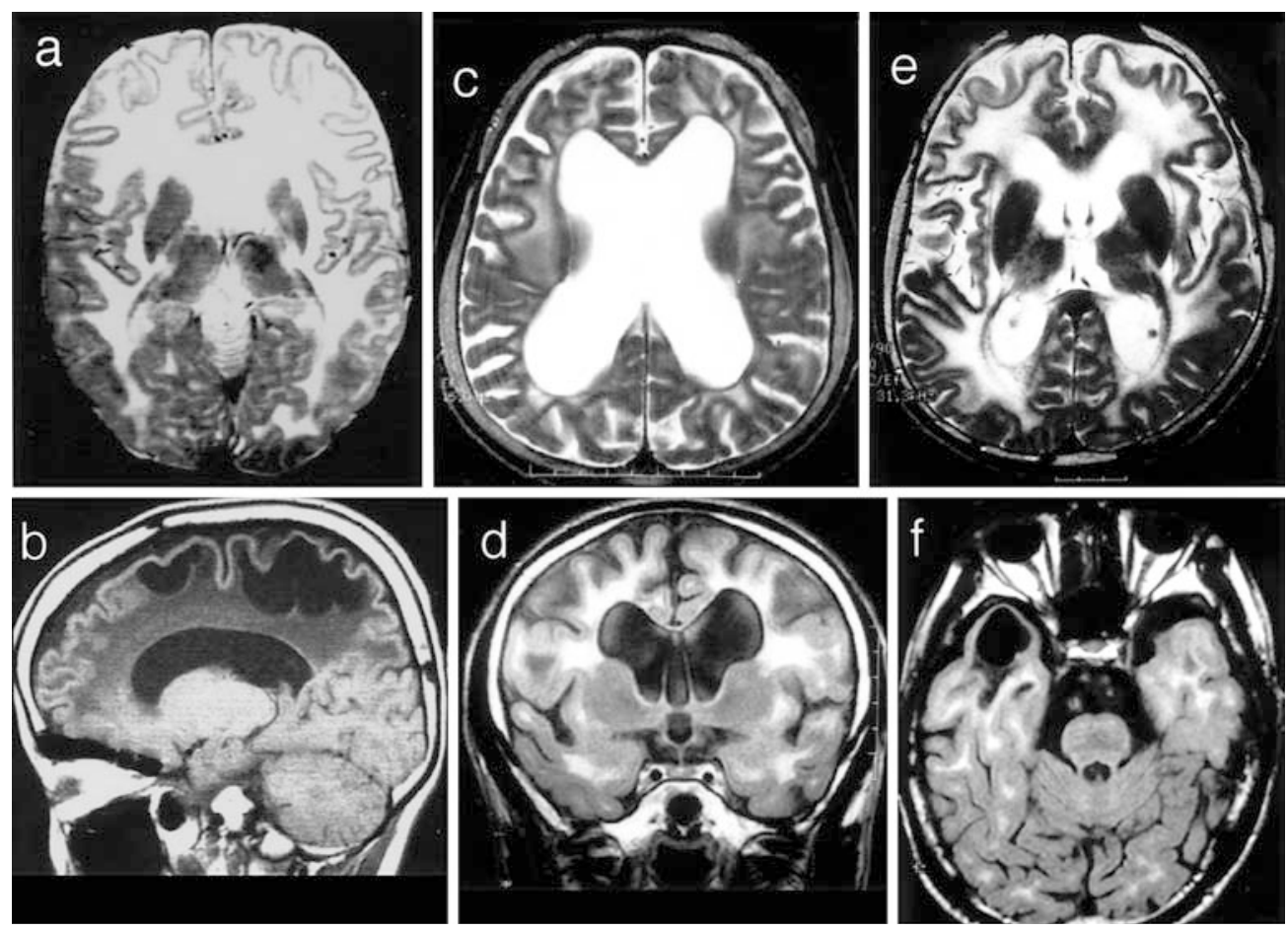

RT-PCR using primers 3 and 4 showed both a cleaved fragment (with S93L) and an uncleaved fragment (with $452-468 \mathrm{del}+\mathrm{g}$ ), but the latter was much thinner than the former, implying that the amount of the mRNA

a

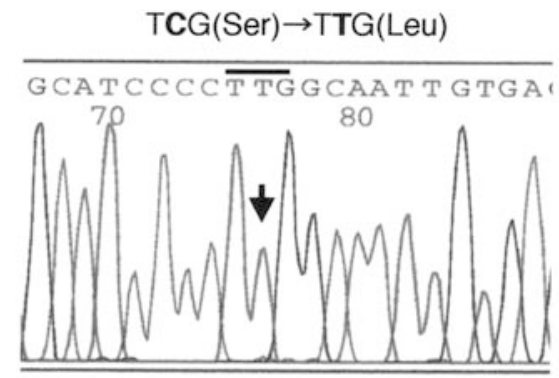

b

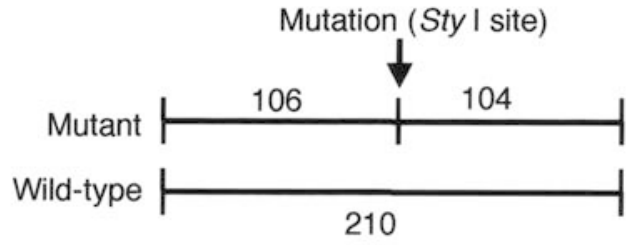

C

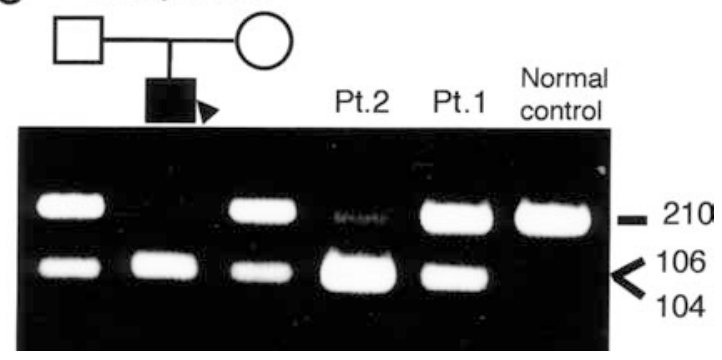

containing $452-468 \mathrm{del}+\mathrm{g}$ is reduced, probably because it is unstable (Fig. 3b, c).

\section{Discussion}

In this study, we analyzed the $M L C 1$ gene in three newly identified Japanese patients. Two of them were homozygous for a previously-described mutation, S93L, and one was a compound heterozygote, with the S93L mutation on one allele and a novel mutation, 452$468 \mathrm{del}+\mathrm{g}$, which leads to a frameshift with a premature termination codon on the other allele. Together with results from a previous report (Leegwater et al. 2001) that described three Japanese patients who had three different mutations, S93L, S280L, and IVS11-2A > G, and with our previous report (Saijo et al. 2003) describing one Japanese patient who was homozygous for $\mathrm{S} 93 \mathrm{~L}$, molecular genetic basis for this disorder has been established for a total of seven Japanese patients. In summary, S93L was observed six of seven $(85.7 \%)$ patients at least in one allele, and ten of $14(71.4 \%)$ alleles had this mutation. Therefore, S93L appears to be fairly frequent in Japanese patients with van der Knaap

Fig. 2a-c Analysis for the S93L mutation. a Sequence of the $M L C 1$ gene of patient 3 showing a C-to-T transition (S93L, arrow). b Representation of segments of the mutant PCR fragment and of the wild-type PCR fragment digested with Sty I. The numbers indicate segment lengths in base pairs. c Pedigree of patient 3 (Pt. 3) and electrophoresis on $3.0 \%$ NuSieve agarose of genomic PCR products of all patients (Pt. 1, patient 1; Pt. 2, patient 2) after digestion with Sty I. Fragment sizes (base pairs) are shown on the right 


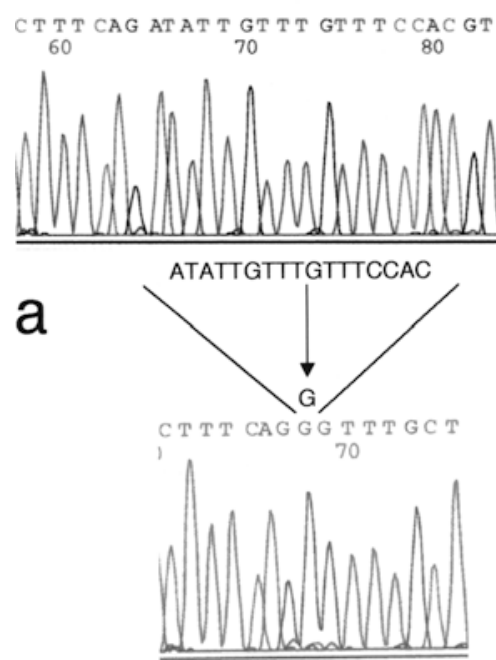

Fig. 3a-c Analysis for the 452-468del + g mutation. a Sequence of a genomic PCR fragment specific for the allele without S93L of a control (upper) and of patient 1 (lower), showing 452-468del + g. b Representation of Sty I-digested segments of RT-PCR fragments (fragment 1) from the wild-type, the allele with 452-468del $+\mathrm{g}$, and the allele with S93L. The numbers indicate segment lengths in base pairs. c Electrophoresis on $1.5 \%$ agarose of RT-PCR fragments of patient 1 and a control after digestion with Sty I. Note that the uncleaved band of patient 1 corresponds to the fragment from the allele with $452-468 \mathrm{del}+\mathrm{g}$. Fragment sizes (base pairs) are shown on both sides

disease, and analysis for this mutation in DNA isolated from leukocytes would provide for an easy and precise diagnosis of this disorder in Japanese patients.

Although all the previous studies on the $M L C 1$ gene used the genomic DNA from patients, we succeeded in effectively amplifying the $M L C 1$ cDNA by RT-PCR from RNA extracted from leukocytes of patients in this study as well as a previous report (Saijo et al. 2003). While the protein encoded by the MLC1 gene may function mainly in the central nervous system, our results indicate that it is expressed in peripheral leukocytes at least sufficiently for RT-PCR. Since the $M L C 1$ gene has 12 exons, our method using the cDNA simplifies the sequencing procedures as compared to the using genomic DNA.

While most patients who have been reported were below age 20 (van der Knaap et al. 1995; Topçu et al. 1998; Yalçinkaya et al. 2000; Ben-Zeev et al. 2001), the patients we describe are older: 23, 26, and 32 years for patients 1,2 , and 3 in this report, and 41 years for the patient we had previously reported (Saijo et al. 2003). This implies that, with appropriate care, patients with van der Knaap disease may have a good prognosis.

Acknowledgements We thank Dr. Fumihiko Sakai and Dr. Toyokazu Saito (Kitasato University) for their support, Dr. Naohide Shiroma (Ryukyu University) for useful discussion, Ms. Kazuko Endate for technical assistance, and Dr. Sara Shanske (Columbia University) for reviewing the manuscript.
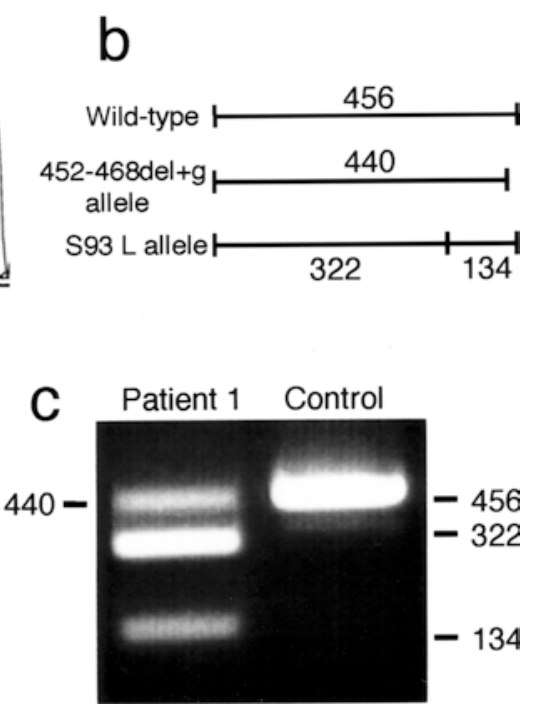

\section{References}

Ben-Zeev B, Gross V, Kushnir T, Shalev R, Hoffman C, Shinar Y, Pras E, Brand N (2001) Vacuolating megalencephalic leukoencephalopathy in 12 Israeli patients. J Child Neurol 16:9399

Leegwater PAJ, Yuan BQ, van der Steen J, Mulders J, Könst AAM, Ilja Boor PK, Mejaski-Bosnjak V, van der Maarel SM, Frants RR, Oudejans CBM, Schutgens RBH, Pronk JC, van der Knaap MS (2001) Mutations of MLC1 (KIAA0027), encoding a putative membrane protein, cause megalencephalic leukoencephalopathy with subcortical cysts. Am J Hum Genet 68:831-838

Saijo H, Nakayama H, Ezoe T, Araki K, Sone S, Hamaguchi H, Suzuki H, Shiroma N, Kanazawa N, Tsujino S, Hirayama Y, Arima M (2003) A case of megalencephalic leukoencephalopathy with subcortical cysts (van der Knaap disease): molecular genetic study. Brain Dev 25:362-366

Topçu M, Saatci I, Topcuoglu MA, Kose G, Kunake B (1998) Megalencephaly and leukodystrophy with mild clinical course: a report on 12 new cases. Brain Dev 20:142-153

Topçu M, Gartioux C, Ribierre F, Yalçinkaya C, Tokus E, Oztekin N, Beckmann JS, Ozguc M, Seboun E (2000) Vacuoliting megalencephalic leukoencephalopathy with subcortical cysts, mapped to chromosome $22 q_{\text {tel }}$. Am J Hum Genet 66:733-739

van der Knaap MS, Barth PG, Stroink H, van Nieuwenhuizen O, Arts WFM, Hoogenraad F, Valk J (1995) Leukoencephalopathy with swelling and a discrepantly mild clinical course in eight children. Ann Neurol 37:324-334

van der Knaap MS, Barth PG, Vrensen GFJM, Valk J (1996) Histopathology of an infantile-onset spongiform leukoencephalopathy with a discrepantly mild clinical course. Acta Neuropathol 92:206-212

Yalçinkaya C, Çomu S, Koçer N, Yuksel A, Gunduz E, Ocal A (2000) Siblings with cystic leukoencephalopathy and megalencephaly. J Child Neurol 15:690-693 